\title{
Pseudomonas endophthalmitis associated with scleral necrosis
}

\author{
K. H. TARR AND I. J. CONSTABLE \\ From the Department of Ophthalmology, Royal Perth Hospital, Perth, Australia
}

SUMMARY Three cases of pseudomonas endophthalmitis associated with scleral necrosis 3 to 10 years after pterygium excision and beta irradiation are described. The value of prophylactic repair of radiation-induced scleral necrosis and early aggressive management of pseudomonas endophthalmitis are emphasised.

Pterygium recurrence is frequent after simple excision alone, especially in hot dry climates. ${ }^{1}$ Beta irradiation is the only treatment that considerably reduces the recurrence rate, and it is frequently used after excision of primary pterygia. ${ }^{2}$ Complications of beta irradiation for this nonmalignant lesion have received little attention. Scleral necrosis from beta irradiation after pterygium excision was first described by Jones and Reese ${ }^{3}$ and subsequently by Cameron, ${ }^{4}{ }^{5}$ Cappin, ${ }^{6}$ and Talbot. ${ }^{7}$ This complication may occur years after beta irradiation and leads to a chronically irritable eye.

Pseudomonas endophthalmitis in such eyes has been reported only once. ${ }^{4}$ This paper reports 3 cases seen over 1 year and emphasises the importance of surgical repair of radiation-induced scleral ulceration.

\section{Case reports}

CASE 1

A 56-year-old European man had bilateral nasal pterygia excised leaving bare sclera in 1968. A total dose of 4000 rads of beta irradiation was applied to each eye over 2 weeks in 3 doses beginning on the 4 th postoperative day. An oval strontium-90 applicator with an emission rate of 870 rads min $^{-1}$ and an active area of $18 \times 13 \mathrm{~mm}$ was used.

The patient was asymptomatic until 1978, when the left eye became red and painful 3 days after it had been hit by a block of wood. On admission the visual acuity was counting fingers. The eye was hyperaemic with a copious mucopurulent discharge. A large necrotic scleral ulcer $6 \mathrm{~mm}$ in diameter

Correspondence to Dr K. H. Tarr, Department of Ophthalmology, Royal Perth Hospital, GPO Box X2213, Perth 6001, Western Australia. with a greenish base was present (Fig. 1) together with a large corneal infiltrate, a fibrinous iridocyclitis, a $1 \mathrm{~mm}$ hypopyon, and vitreous condensation. A heavy growth of Pseudomonas aeruginosa was cultured from the ulcer base, and diminishing numbers could be isolated until 21 days after admission. All were pyocin type UT/a.

Hourly gentamicin drops and intramuscular tobramycin (120 mg 8 hourly) were given for 3 days, but the eye deteriorated. Therefore treatment was changed to intravenous ticarcillin via a superior vena cava catheter $(4 \mathrm{~g}$ in $20 \mathrm{ml}$ normal saline infused over 10 minutes every 4 hours, that is, 24 $\mathrm{g} /$ day or $300 \mathrm{mg} / \mathrm{kg} /$ day) for 6 weeks together with oral probenecid $2 \mathrm{~g}$ daily. Tobramycin was given by subconjunctival injection (10 mg daily) for 2 weeks by continuous infusion into the upper fornix (320 mg $\mathrm{l}^{-1}$ normal saline at $0.3 \mathrm{ml} \mathrm{min}^{-1}$ ) for the second 2 weeks and then by oculentum tobramycin

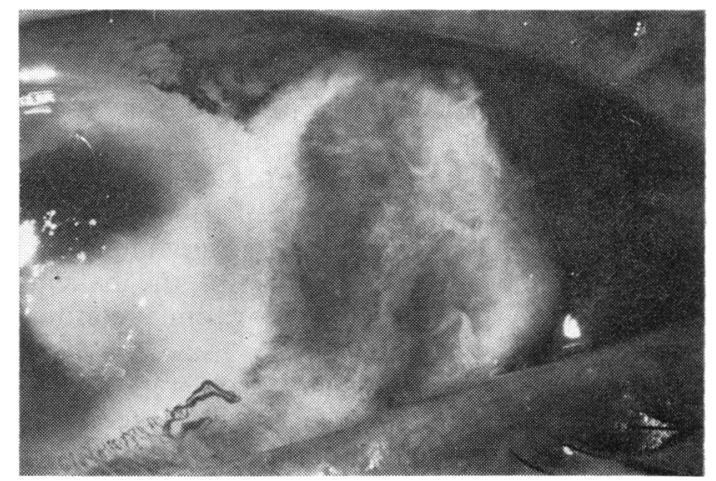

Fig. 1 Case 1: left eye, large necrotic scleral ulcer with endophthalmitis 10 years after pterygium excision and beta irradiation. 


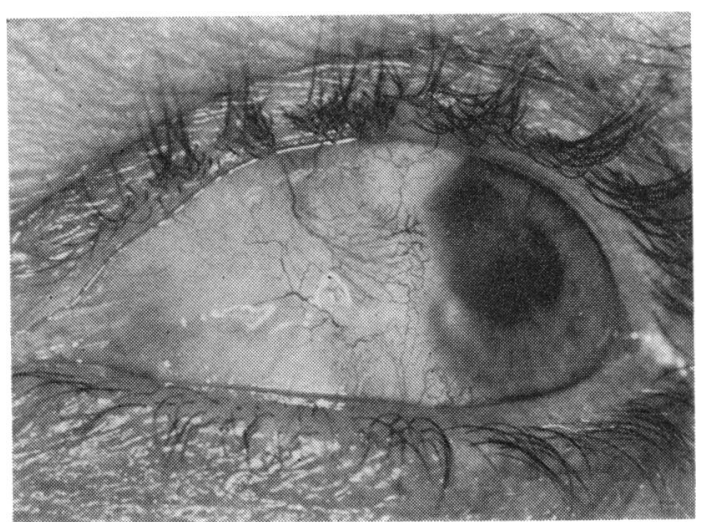

Fig. 2 Case 1: left eye, 1 year after a conjunctival flap.

$0.3 \% 6$ times a day for 2 weeks. Six weeks after the patient's admission to hospital a conjunctival flap was placed over the ulcer, and antibiotics ceased 1 week later.

The right visual acuity was $6 / 5$. An avascular thin cystic conjunctiva extended from 2 to 4 o'clock surrounded by tufts of episcleral vessels. The subjacent sclera was porcelain white. One year later the left visual acuity was 6/9. The sclera was well covered by conjunctiva. A superficial corneal opacity, posterior synechiae, moderate lens opacities, and membranous vitreous opacities were present (Fig. 2).

Early admission to hospital and intense and prolonged treatment were important factors in salvaging this eye. The ulcer site and size indicated that radiation-induced scleral pathology predisposed to the subsequent ulceration and infection.

\section{CASE 2}

A European man now aged 70 years had a left nasal pterygium excised leaving bare sclera in 1968. A total dose of 2800 rads of beta irradiation was given in 2 applications on the 6 th and 11 th days postoperatively. The source was the same as in case 1 .

The eye was asymptomatic until 1978, when he presented to a rural hospital with a painful red eye. A history of trauma was denied. Pseudomonas aeruginosa was cultured from a conjunctival swab, and as the eye deteriorated he was transferred 1 week later. On examination there was a severe hyperaemia and a purulent discharge. Medially a large necrotic ulcer extended $2 \mathrm{~mm}$ on to cornea and $4 \mathrm{~mm}$ on to sclera. A corneal infiltrate adjacent to the ulcer and a $2 \mathrm{~mm}$ hypopyon were present. Pseudomonas was again cultured from conjunctival swabs. Despite gentamicin topically every hour and intramuscularly (180 $\mathrm{mg} /$ day $)$ and carbenicillin intravenously ( $30 \mathrm{~g} /$ day) the eye deteriorated, with extension of the scleral necrosis. The eye was eviscerated 3 weeks after the infection began.

The site and appearance of the ulceration indicated that radiation-induced necrosis predisposed the eye to pseudomonas endophthalmitis. The delay in starting treatment contributed to loss of the eye.

\section{CASE 3}

A 57-year-old Aboriginal woman had bilateral nasal pterygia excised leaving bare sclera in 1976. A total dose of 2400 rads of beta irradiation was applied over 2 weeks in 3 doses beginning on the day of operation. The applicator and dose rate were similar to that described above.

In September 1978 she presented with a 2-week history of a red painful photophobic eye. The visual acuity was right $6 / 12$ and left 6/36. The reduced vision was due to lens opacities. Scleral necrosis was present medially in each eye, $2 \mathrm{~mm}$ from the limbus and $2 \mathrm{~mm}$ in diameter. The ulceration was almost full thickness with a grey-brown base composed of a thin layer of scleral fibres. The left eye was hyperaemic, with an iridocyclitis and corneal oedema. No organisms were cultured from conjunctival swabs. The eye settled with topical treatment, and 3 weeks later the left ulcer was closed with a scleral graft and conjunctival flap.

In June 1979 the patient was readmitted with a 1-week history of a right conjunctivitis progressing despite topical chloramphenicol and systemic amoxycillin. There was no history of trauma. The right eye had light perception only, gross periorbital oedema, marked conjunctival hyperaemia, corneal oedema, and a $360^{\circ}$ peripheral ring hypoyon. The scleral ulceration was unchanged (Fig. 3).

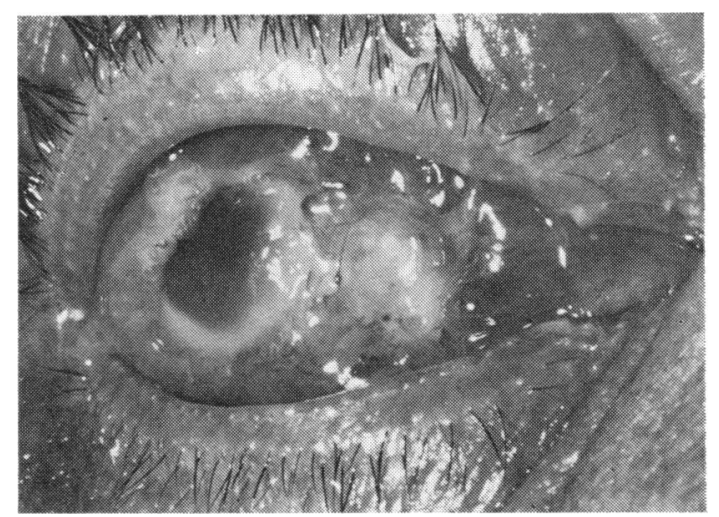

Fig. 3 Case 3: right eye, scleral ulceration with endophthalmitis 3 years after pterygium excision and beta irradiation. 
Pseudomonas aeruginosa was repeatedly cultured from conjunctival swabs. Despite systemic gentamycin (120 mg daily) and carbenicillin ( $2 \mathrm{~g}$ daily) and topical gentamycin and chloramphenicol the condition of the eye worsened, and it was eviscerated 8 days after admission. The left eye had healed well (Fig. 4).

The bilateral nature of the deep scleral ulceration, the successful outcome of the operated eye, and the subsequent pseudomonas endophthalmitis and loss of the fellow eye serve to emphasise the importance of repairing scleral ulceration after beta irradiation.

\section{Discussion}

Pseudomonas aeruginosa is widely distributed in nature and can be found in water, soil, waste, and as a commensal of the intestinal tract and skin. The commonest ocular infection is a hypopyon corneal ulcer or abscess. Prior epithelial damage is often present. Rarely it causes postoperative endophthalmitis, lid infection, primary conjunctivitis, dacryocystitis, or orbital cellulitis. ${ }^{8}$ It is a classical opportunist, and infection particularly arises in patients predisposed by defective immunity or altered commensal flora by broad-spectrum antibiotics.

Radiation-induced scleral necrosis is an uncommon but an increasingly recognised complication of pterygium management. The above cases are from a group of over 40 patients seen recently in Western Australia with scleral ulceration. No longer can the sclera be considered resistant to radiation necrosis even after modest dosages of beta irradiation.

Infection in eyes with scleral disease has not been previously emphasised. No mention has been made of bacterial endophthalmitis as a complication of necrotising scleritis. ${ }^{10}$ However, pseudomonas endophthalmitis is a significant complication of beta irradiation-induced scleral ulceration. It occurred in one of 6 cases reported by Cameron. ${ }^{4}$ Talbot $^{7}$ reported 13 cases of conjunctival and scleral ulceration of which two developed 'perforation, uveitis and blindness', but there is no mention of infection. Our cases indicate that pseudomonas may arise spontaneously in eyes with scleral ulceration even when this is only partial thickness. Such defects therefore should be repaired by a conjunctival flap or by a graft of sclera, cornea, fascia lata, or other tissue to prevent this devastating infection.

There is no ready explanation for the occurrence of pseudomonas infection in irradiation-induced scleral disease but apparently not in necrotising scleritis. Certainly scleral destruction is more extensive after scleritis than irradiation. Suppression of the immunological apparatus by $x$-irradiation is well known. However, experimentally Silverstein ${ }^{11}$

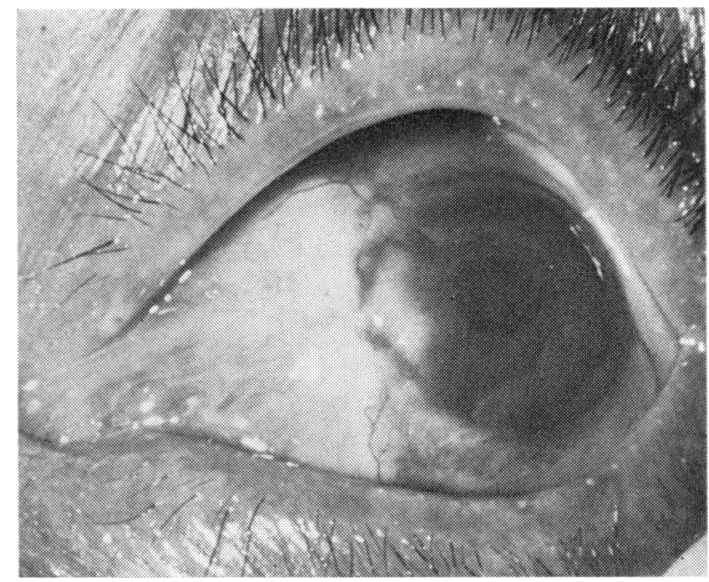

Fig. 4 Case 3: left eye, 9 months after a scleral graft and conjunctival flap.

has clearly shown that ocular irradiation has no effect on the activation of the immune system towards foreign antigens induced into the eye.

The large scleral ulcerations in cases 1 and 2 are atypical of beta irradiation-induced ulcers, which usually do not involve the cornea and are rarely as extensive. Most probably pseudomonas proteases extended the ulceration.

Salvage of one eye must be attributed to early, prolonged, and intensive antibiotic treatment. Persistence of pseudomonas organisms despite treatment and recrudescence of the infection after treatment is ended has been well described ${ }^{12}$ and is an important factor in the management of pseudomonas infections. Prolonged treatment is therefore essential, and a continuous local delivery system is advocated. ${ }^{12}$ This not only delivers the antibiotic continuously with minimal discomfort but also washes away pus, debris, and pseudomonas proteases. Antibiotic effectiveness is enhanced and further destruction of ocular tissue is minimised. The alternative-repeated subconjunctival injections-is extremely painful and irritative, so that the number of repeated injections is limited.

Synergism between ticarcillin and tobramycin has been demonstrated in vitro and in vivo. ${ }^{13}$ Adequate aqueous levels of tobramycin occur with subconjunctival and topical use. ${ }^{14}$ Ticarcillin is more effective than carbenicillin against pseudomonas. ${ }^{15}$ When pseudomonas organisms resistant to gentamicin or carbenicillin are present, or when the clinical response is inadequate, the combination of ticarcillin and tobramycin should be used.

We are most grateful to Drs G. Bougher, I. North, and T. M. Wilson for permission to study their patients. We also thank Dr A. J. M. Nelson for providing radiotherapy details. 


\section{References}

'Duke-Elder S. System of Ophthalmology. London: Kimpton, 1965: 8: 574.

'Cameron ME. Pterygium Throughout the World. Illinois: Thomas, 1965: 141-3.

'Jones IS, Reese AB. Focal scleral necrosis. Arch Ophthalmol 1953; 49: 633-6.

Cameron ME. Preventable complications of pterygium excision with beta irradiation. $\mathrm{Br} J$ Ophthalmol 1972; 56: 52-6.

${ }^{5}$ Cameron ME. The treatment of beta irradiation necrosis of sclera. Aust J Ophthalmol 1978; 6: 86-9.

-Cappin JM. Radiation scleral necrosis simulating early scleromalacia perforans. Br J Ophthalmol 1973; 57: 425-8.

'Talbot AN. Complications of beta ray treatment of pterygia. Trans Ophthalmol Soc NZ 1979; 31: 62-3.

'Gutierrez EH. In: Locatcher-Khorazo D, Seegal, BC, eds. Microbiology of the Eye. London: Mosby, Kimpton, 1972: 64-79.
${ }^{9}$ Watson PG, Hayreh SS. Scleritis and episcleritis. $\mathrm{Br} J$ Ophthalmol 1976; 60: 163-91.

${ }^{10}$ Fraunfelder FT, Watson PG. Evaluation of eyes enucleated for scleritis. Br J Ophthalmol 1976; 60: 227-30.

${ }^{11}$ Silverstein AM. Immunogenic uveitis. Trans Ophthalmo! Soc UK 1974; 94: 496-517.

${ }^{12}$ Hessburg PC. Treatment of pseudomonas keratitis in humans. Am J Ophthalmol 1966; 61 : 896-903.

${ }^{13}$ Comber KR, Basker MJ, Osborne CD, Sutherland $R$. Synergy between ticarcillin and tobramycin against Pseudomonas aeruginosa and Enterobacteriaceae in vitro and in vivo. Antimicrob Agents Chemother 1977; 11: 956-64.

${ }^{14}$ Davis SD, Sariff LD, Hyndiuk RA. Antibiotic therapy of experimental pseudomonas keratitis in guinea pigs. Arch Ophthalmol 1977; 95: 1638-43.

${ }^{15}$ Prior RB, Fass RJ. Comparison of ticarcillin and carbenicillin activity against random and select populations of Pseudomonas aeruginosa. Antimicrob Agents Chemother 1978; 13: 184-7. 